\title{
Calculating time dilation using Euler's formula
}

\author{
Andrea Conte ${ }^{1, *}$
}

\begin{abstract}
This paper shows how the time dilation due to motion, calculated normally using the Lorentz factor, can be encoded in the real part of a complex number by using the Euler's formula. The imaginary part of this complex number will contain the velocity. It also shows how the time dilation due to gravitational attraction can be encoded using the same formula. A combination of time dilation and gravitational time dilation is presented using this formula. The magnitude of this complex number represents the constancy of the speed of light.
\end{abstract}

Keywords: Time dilation, Euler's formula, Complex number, Special Relativity, Relativity, Lorentz Factor, Combination time dilation

\footnotetext{
*Corresponding author

Email address: conte.an@hotmail.com (Andrea Conte)

${ }^{1}$ Declarations of interest: none
} 


\section{Contents}

1 Introduction 3

2 Encoding time dilation due to motion using Euler's formula 4

3 Encoding time dilation due to gravitational attraction using Euler's formula 5

4 Combination of time dilation and gravitational time dilation using Euler's $\begin{array}{lr}\text { formula } & 6\end{array}$

5 Speed of light as the magnitude of Euler's formula $\quad 8$

6 Conclusion $\quad 8$

$\begin{array}{llll}\text { Appendix } & \text { A } & \text { Supplementary data } & 8\end{array}$ 


\section{Introduction}

The Lorentz's factor Eq.(1) and Eq.(2), is the time dilation equation used to calculate the time dilation of a system in motion while the gravitational time dilation equation Eq.(3) is the equation used to calculate the time dilation due to the gravitational attraction $[2,3,4]$.

$$
\begin{aligned}
& \text { Standard form of Lorentz factor }=\frac{1}{\sqrt{1-\frac{v^{2}}{c^{2}}}} \\
& \text { Inverse form of Lorentz factor }=\frac{\sqrt{1-\frac{v^{2}}{c^{2}}}}{1} \\
& \text { Gravitational time dilation }=\sqrt{1-\frac{2 G M}{r c^{2}}}
\end{aligned}
$$

Eq.(1) is the standard form of Lorentz factor in which the number 1 represents the time in seconds on an observer's clock inside a system in motion. While the speed v increases compared to an observer at rest outside the system, the factor will increase over 1 and will represent the time that has passed outside the system while 1 second has elapsed inside the system in motion. If the system is in motion at the speed $4 / 5$ of the speed of light, the time of the outside observer's clock will show $\approx 1.5$ seconds when the clock inside the system in motion will have reached only 1 second. This means that the outside observer's clock (at rest) will run faster. The number 1 is called the proper time of the inside observer's clock (in motion).

Eq.(2) is the inverse form of Lorentz factor. It encodes the same result and meaning but on the opposite way. When 1 second has been reached on the outside observer's clock at rest, then only $\approx 0.66$ seconds will have elapsed inside the system in motion using the inside observer clock. Compare to the standard form, the number 1 in the inverse form represents the proper time of the outside observer at rest. It is easy to verify that $1 \mathrm{~s} / 0.66 \mathrm{~s} \approx 1.5 \mathrm{~s}$ meaning the outside observer's clock is 1.5 times faster than the inside observer's clock. The inverse form is the preferred form used in this paper because this is the form obtained by using the Euler's formula to encode time dilation as presented in section 2 .

The Euler's formula is presented in Eq.(4). The symbol e represents the Euler's number. The symbol i represents the imaginary unit number, $\theta$ represents an angle in radians. The result gives the complex number with a real part of cosine of $\theta$ and the imaginary part of i multiplied by sine of $\theta$.

$$
e^{i \theta}=\cos (\theta)+i \sin (\theta)
$$


The next sections present methods of encoding the time dilation, due to motion and due to gravitational attraction using the Euler's formula. To obtain this encoding, the only value modified in the the Euler's formula to obtain the time dilation will be the variable $\theta$ which represents the argument of the complex number.

\section{Encoding time dilation due to motion using Euler's formula}

In Eq.(4), when $\theta$ is chosen to be $\arcsin (\alpha)$, with $\alpha$ equals to $v / c$, the resulting complex number gives the Lorentz factor inverse in its real part and the speed $v / c$ in its imaginary part. the value $\arcsin (v / c)$ is included between 0 and $\pi / 2$ and $\mathrm{v} / \mathrm{c}$ is included between 0 and 1 inclusive. The followings equations sum up these properties.

$$
\begin{aligned}
& \alpha=\frac{v}{c} \\
& 0 \leq \alpha \leq 1 \\
& 0 \leq \arcsin (\alpha) \leq \pi / 2 \\
& e^{i \arcsin (\alpha)}=\cos (\arcsin (\alpha))+i \sin (\text { arcsin }(\alpha)) \\
& \cos (\arcsin (\alpha))=\sqrt{1-\frac{v^{2}}{c^{2}}}=\text { Lorentz factor inverse } \\
& e^{i \arcsin (\alpha)}=\sqrt{1-\frac{v^{2}}{c^{2}}+i \alpha} \\
& \left.i \sin (\arcsin (\alpha))=i \alpha=i \frac{v}{c}=\text { speed (in speed of light unit }\right)
\end{aligned}
$$

To obtain only the real part that encodes the time dilation, the following equation can be used in which $\theta$ represents $\arcsin (\alpha)$. This equation will set the imaginary part to 0 .

$$
\cos (\theta)=\frac{e^{i \theta}+e^{-i \theta}}{2}=\sqrt{1-\frac{v^{2}}{c^{2}}}
$$


Inverting this equation gives you the standard form of the Lorentz factor.

$$
\cos (\theta)=\frac{2}{e^{i \theta}+e^{-i \theta}}=\frac{1}{\sqrt{1-\frac{v^{2}}{c^{2}}}}
$$

To obtain only the imaginary part that encodes the speed, the following equation can be used in which $\theta$ represents $\arcsin (\alpha)$. This equation will set the real part to 0 .

$$
\sin (\theta)=\frac{e^{i \theta}-e^{-i \theta}}{2}=i \alpha=i \frac{v}{c}
$$

\section{Encoding time dilation due to gravitational attraction using Euler's formula}

To encode time dilation due to gravitational attraction, the value $\alpha$ must be $\sqrt{2 G M / r c^{2}}$. The value is obtained using the mechanical energy equation of a satellite when the mechanical energy is set to zero.

Kinetic Energy - Potential Energy = Mechanical energy

Kinetic Energy - Potential Energy $=0$

Kinetic Energy = Potential Energy

$$
\begin{aligned}
\frac{1}{2} m v^{2} & =G \frac{M m}{r} \\
m v^{2} & =2 G \frac{M m}{r} \\
v & =\sqrt{2 G \frac{M}{r}}
\end{aligned}
$$

The final step is to divide both side by $c$.

$$
\begin{aligned}
& \frac{v}{c}=\frac{\sqrt{2 G \frac{M}{r}}}{c} \\
& \frac{v}{c}=\sqrt{2 G \frac{M}{r c^{2}}}
\end{aligned}
$$

This last equation represents an equivalence between $v / c$ and $\sqrt{2 G M / r c^{2}}$. Both can be used in place of $\alpha$ in the $\arcsin (\alpha)$ value. The followings equations sum up the properties for the gravitational time dilation.

$$
\begin{aligned}
& \alpha=\sqrt{2 G M / r c^{2}} \\
& 0 \leq \alpha \leq 1
\end{aligned}
$$




$$
\begin{aligned}
& 0 \leq \arcsin (\alpha) \leq \pi / 2 \\
& e^{i \arcsin (\alpha)}=\cos (\arcsin (\alpha))+i \sin (\arcsin (\alpha)) \\
& \cos (\arcsin (\alpha))=\sqrt{1-\frac{2 G M}{r c^{2}}}=\text { Gravitational time dilation } \\
& i \sin (\arcsin (\alpha))=i \alpha=i \sqrt{2 G M / r c^{2}}=i \frac{v}{c} \\
& e^{i \arcsin (\alpha)}=\sqrt{1-\frac{2 G M}{r c^{2}}}+i \alpha
\end{aligned}
$$

To obtain only the real part that encodes the time dilation, the following equation can be used in which $\theta$ represents $\arcsin (\alpha)$. This equation will set the imaginary part to 0 .

$$
\cos (\theta)=\frac{e^{i \theta}+e^{-i \theta}}{2}=\sqrt{1-\frac{2 G M}{r c^{2}}}
$$

To obtain only the imaginary part that encodes the speed, the following equation can be used in which $\theta$ represents $\arcsin (\alpha)$. This equation will set the real part to 0 .

$$
\sin (\theta)=\frac{e^{i \theta}-e^{-i \theta}}{2}=i \alpha=i \sqrt{2 G M / r c^{2}}=i \frac{v}{c}
$$

\section{Combination of time dilation and gravitational time dilation using Euler's formula}

To combine time dilation and gravitational time dilation using the official equations, Eq.(26) can be used. The time dilation is represented by the symbol $\gamma$.

$$
\gamma=\left(\frac{\sqrt{1-\frac{v_{1}^{2}}{c^{2}}}}{\sqrt{1-\frac{v_{2}^{2}}{c^{2}}}}\right) *\left(\frac{\sqrt{1-\frac{2 G M}{r_{1} c^{2}}}}{\sqrt{1-\frac{2 G M}{r_{2} c^{2}}}}\right)
$$

The top part represent a system in one frame of reference while the bottom part represents a system in another frame of reference. In the top parts, $v_{1}$ represents the speed 
of the first system and the distance $r_{1}$ represents the distance of the first system from the center of mass M. In the bottom parts, $v_{2}$ represents the speed of the second system and the distance $r_{2}$ represents the distance of the second system from the center of mass $\mathrm{M}$.

When $\gamma<1$, then the system represented by the top part is slower than the system represented by the bottom part. When $\gamma>1$, it means the system at the top is faster.

For example, given $v_{1}=3889, r_{1}=26378000, v_{2}=460$ and $r_{2}=6378000$, the result will be 1.000000000444252 . The values $v_{1}$ and $r_{1}$ corresponds to a satellite's clock around the earth and $v_{2}$ and $r_{2}$ corresponds to a clock on the ground at rest. The satellite's clock will go faster of about 38 microseconds/day [1,5]. When $1.000000000444252 \mathrm{~s}$ has elapsed on the satellite's clock, only $1 \mathrm{~s}$ has elapsed on the ground's clock.

$$
\gamma=\frac{\sqrt{1-\frac{v_{1}^{2}}{c^{2}}}}{\sqrt{1-\frac{v_{2}^{2}}{c^{2}}}} * \frac{\sqrt{1-\frac{2 G M}{r_{1} c^{2}}}}{\sqrt{1-\frac{2 G M}{r_{2} c^{2}}}}=\frac{1.000000000444252}{1}
$$

86400 seconds $/$ day $*(1.000000000444252-1)=+0.000038383$ seconds $/$ day

$$
=+38 \text { microseconds/day }
$$

To combine time dilation and gravitational time dilation using Euler's formula, the following equation can be used. Putting the same values of $v_{1}, r_{1}, v_{2}$ and $r_{2}$ as in Eq.(27), the result $\gamma$ will be the same.

$$
\begin{gathered}
\gamma=\left(\frac{e^{i \arcsin \left(\alpha_{1}\right)}+e^{-i \arcsin \left(\alpha_{1}\right)}}{e^{i \arcsin \left(\alpha_{2}\right)}+e^{-i \arcsin \left(\alpha_{2}\right)}}\right) *\left(\frac{e^{i \arcsin \left(\alpha_{3}\right)}+e^{-i \arcsin \left(\alpha_{3}\right)}}{e^{i \arcsin \left(\alpha_{4}\right)}+e^{-i \arcsin \left(\alpha_{4}\right)}}\right) \\
\Rightarrow \quad \alpha_{1}=\frac{v_{1}}{c}, \quad \alpha_{2}=\frac{v_{2}}{c}, \quad \alpha_{3}=\sqrt{2 G \frac{M}{r_{1} c^{2}}}, \quad \alpha_{4}=\sqrt{2 G \frac{M}{r_{2} c^{2}}}
\end{gathered}
$$

The following equation is an alternative derived from Eq.(28).

$$
\begin{array}{r}
\gamma=\left(e^{-i(\arcsin (\alpha 1)-\arcsin (\alpha 2)+\arcsin (\alpha 3)-\arcsin (\alpha 4))}\right) \\
* \frac{\left(1+e^{2 i \arcsin (\alpha 1)}\right)\left(1+e^{2 i \arcsin (\alpha 3)}\right)}{\left(1+e^{2 i \arcsin (\alpha 2)}\right)\left(1+e^{2 i \arcsin (\alpha 4)}\right)}
\end{array}
$$




\section{Speed of light as the magnitude of Euler's formula}

Whatever value you choose for $\theta$, the magnitude of the complex number $e^{i \theta}$ is 1 . In this context, when $\theta$ is replaced by $\arcsin (v / c)$, it represents the constant speed of light $\mathrm{c}$ with a unit of 1 .

$$
\left|e^{i \theta}\right|=1=\sqrt{(\cos (\theta))^{2}+(i \sin (\theta))^{2}}
$$

To represent the speed of light in $\mathrm{m} / \mathrm{s}$ namely $299792458 \mathrm{~m} / \mathrm{s}$, it is sufficient to add the value of the speed of light in $\mathrm{m} / \mathrm{s}$ as a multiplicative term in front of $e$.

$$
\left|c e^{i \theta}\right|=\left|299792458 e^{i \theta}\right|=299792458
$$

The magnitude value, representing the speed of light, will stay constant whatever value you choose for $\mathrm{v}$ in $\arcsin (\mathrm{v} / \mathrm{c})$.

\section{Conclusion}

An encoding of time dilation using Euler's formula has been presented. This encoding can be seen as a conversion from the real domain to the complex domain. One characteristic of this encoding is that the $v$ and $\sqrt{2 G M / r}$ are interchangeable and can encode the same intensity of time dilation when chosen properly. This also show that time dilation due to motion and due to gravitational can be made equivalent in value. A combination of time dilation and gravitational time dilation was also presented.

\section{Appendix A. Supplementary data}

The script to calculate the combination of time dilation and gravitational time dilation using Euler's formula is available on the OSF repository at https://osf .io/n3zgy

\section{References}

[1] Ashby, N., 2003. Relativity in the global positioning system. doi:10.12942/ lrr-2003-1.

[2] Einstein, A., 1905. On the electrodynamics of moving bodies (Zur Elektrodynamik bewegter Körper). Annalen der Physik .

[3] Einstein, A., 1916. The Foundation of the General Theory of Relativity (Die Grundlage der allgemeinen Relativitätstheorie). Annalen der Physik doi:10.1002/andp. 19163540702.

[4] Einstein, A., 1920. Relativity: The Special \& General Theory.

[5] Pogge, R.W., 2017. Real-World Relativity: The GPS Navigation System. URL: https: //bit.1y/2XfWPYD. 